\title{
Exercise in Obesity—the Role of Technology in Health Services: Can This Approach Work?
}

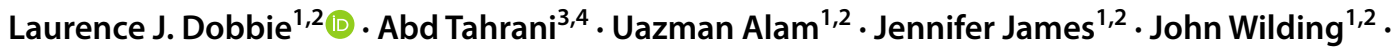 \\ Daniel J. Cuthbertson ${ }^{1,2}$
}

Accepted: 2 September 2021 / Published online: 17 November 2021

(c) The Author(s) 2021

\begin{abstract}
Purpose of Review Physical activity (PA) is an important strategy to prevent and treat obesity. Electronic health (eHealth) interventions, such as wearable activity monitors and smartphone apps, may promote adherence to regular PA and successful weight loss. This review highlights the evidence for eHealth interventions in promoting PA and reducing weight.

Recent Findings Wearables can increase PA and are associated with moderate weight loss in middle/older-aged individuals, with less convincing effects long-term ( $>1$ year) and in younger people. Data for interventions such as mobile phone applications, SMS, and exergaming are less robust. Investigations of all eHealth interventions are often limited by complex, multi-modality study designs, involving concomitant dietary modification, making the independent contribution of each eHealth intervention on body weight challenging to assess.

Summary eHealth interventions may promote PA, thereby contributing to weight loss/weight maintenance; however, further evaluation is required for this approach to be adopted into routine clinical practice.
\end{abstract}

Keywords Obesity · eHealth · Physical activity $\cdot$ Wearables $\cdot$ Exergaming

$\begin{array}{ll}\text { Abbreviations } \\ \text { SBWL } & \text { Standard behavioural weight loss } \\ \text { PA } & \text { Physical activity } \\ \text { WL } & \text { Weight loss } \\ \text { T2D } & \text { Type } 2 \text { diabetes mellitus } \\ \text { NALFD } & \text { Non-alcoholic fatty liver disease } \\ \text { BMI } & \text { Body mass index }\end{array}$

This article is part of the Topical Collection on Health Services and Programs

Daniel J. Cuthbertson

Dan.Cuthbertson@liverpool.ac.uk

Laurence J. Dobbie

Laurence.dobbie@liverpool.ac.uk

1 Department of Cardiovascular and Metabolic Medicine, Institute of Life Course and Medical Sciences, University of Liverpool, Liverpool, UK

2 University Hospital Aintree, Liverpool University Hospitals NHS Foundation Trust, Liverpool, UK

3 Institute of Metabolism and Systems, School of Clinical and Experimental Medicine, University of Birmingham, Birmingham, UK

4 Department of Diabetes and Endocrinology, Birmingham Heartlands Hospital, Birmingham, UK
BCT Behaviour change techniques

RCT Randomised controlled trial

MVPA Moderate-vigorous physical activity

LPA Light physical activity

eHealth Electronic health

\section{Introduction}

The past five decades has witnessed profound and continued evolution of the characteristics and quantities of food that individuals consume (particularly processed and ultraprocessed food) and in the amount of physical activity (PA) which individuals undertake. A chronic mismatch between energy intake, with consumption of caloric excess/energydense foods, and energy expenditure, with increasing rates of physical inactivity, underlies the current obesity epidemic. Insufficient physical activity, also known as physical inactivity (defined as not undertaking at least $150 \mathrm{~min}$ of moderateintensity, or $75 \mathrm{~min}$ of vigorous-intensity PA/week, or any equivalent combination of the two, including PA at work, at home, for transport, and during leisure time) has a global age-standardised prevalence of $27.5 \%$ (95\% uncertainty interval 25.0-32.2) [1]. The prevalence of insufficient PA is 
highest and continually increasing in high-income countries, with women less active than men worldwide. Implementation of targeted evidence-based interventions, presented in the Global Physical Activity Action Plan 2018-2030, and publication of new Guidelines on Physical Activity and Sedentary Behaviour aim to improve population health by reversing the current trends and reducing disparities in physical activity $[2,3]$.

Current intervention strategies for prevention and treatment of obesity have significant limitations. The most effective strategy currently available for treating severe obesity is bariatric/metabolic surgery, but this may be associated with medical complications and weight regain and availability is limited [4]. Moderate-intensity regular PA alone is generally associated with moderate weight loss only, but still encouraging increased PA is an important strategy for optimising the effects of dietary modification and for weight loss maintenance [5-7]. Technology (computers, smartphones, internet usage, video gaming) has become embedded in our society with complex consequences for PA and obesity levels. Yet, ironically some of the same technological advances that have driven reductions in PA may also be adopted to facilitate increased levels of PA (and dietary compliance) for the prevention of obesity, as an effective adjunct to its treatment and in addressing obesity-related morbidities and for successful weight-loss maintenance [8].

\section{Benefits of Physical Activity}

The benefits of physical activity includes preventing or improving many chronic diseases and impacting on both allcause mortality and life expectancy [9-11]. Wen et al. performed a prospective cohort study on 416,175 individuals in Taiwan from 1996 to 2008 to evaluate the impact of different levels of physical activity on all-cause mortality and life expectancy [12]. Individuals were placed into 5 categories: inactive, low, medium, high, and very high activity, according to their weekly exercise volume assessed using a self-administered questionnaire. The data highlighted that those in the low PA group, who exercised for an average of $15 \mathrm{~min} /$ day, had a $14 \%$ reduced risk of all-cause mortality and had a 3-year longer life expectancy, compared with those in the inactive group. Furthermore, each additional $15 \mathrm{~min}$ of exercise/day (beyond the minimum amount of $15 \mathrm{~min} /$ day) reduced all-cause mortality and all-cancer mortality by $4 \%$ and $1 \%$ respectively. These benefits were noted in both sexes, all ages and those with high cardiovascular disease (CVD) risk.

\section{Traditional and Novel Methods of Implementing Exercise Interventions}

Traditionally, exercise interventions have been supervised 'in person' on an individual or group basis; this pattern of intervention delivery is not feasible for large-scale public health interventions. The current coronavirus pandemic has impacted the way in which individuals engage in PA and has seen adoption of novel innovations to facilitate remote supervision and monitoring of PA. Strategies to enhance PA adherence and sustainability are associated with greater benefit. Increasingly, there has been interest in technology-based interventions to promote physical activity and health more remotely which have been shown to be effective in increasing PA levels [13]. These electronic Health (eHealth) interventions represent novel approaches in the promotion of PA, and reduction of body weight, and are vital given that $~ 50 \%$ of individuals attempting weight loss do not engage in any PA [14]. eHealth technologies include wearable devices, social networking sites (SNS), smartphone applications, short messaging service (SMS) and exergaming [13, 15-17]. For eHealth interventions to be implemented within obesity healthcare services, the technologies must be based on clinically proven behaviour change techniques (BCT), which are considered to be the 'active ingredients' in behaviour change interventions, with an increasing evidence base to support their use in the promotion of PA in clinical care [18].

Advances in digital technology and digital platforms can facilitate adherence to behavioural regimes and increase PA levels, optimising the process of self-monitoring (with automation of monitoring and feedback), enhancing self-efficacy and improving the motivation for behaviour change and, therefore, leading to greater success in long-term weight loss and maintenance [19]. Simultaneously, these technologies can be adapted to implement educational interventions and other changes in health behaviour such as dietary modification. Evidence to date suggests that electronic monitoring methods promote higher rates of adherence to self-monitoring than traditional paper-based methods [20-22]. This narrative review shall discuss the various modalities in which technology has been applied to enhance PA and exercise in individuals living with overweight and obesity.

\section{Wearables (Table 1)}

Wearable technologies include smartwatches (e.g. Fitbit, Apple Watch) and provide continuous feedback on PArelevant indices including step count, energy expenditure and heart rate [23]. The data generated facilitates goal setting, objective PA self-monitoring and social support via SMS which are evidence-based BCTs [20, 24, 25]. Automated self-monitoring aids may prompt identification of detrimental behaviour changes and overcomes significant limitations of paper monitoring such as imprecise recall and social desirability bias $[15,19,21,22,26,27]$. Wearables have considerable potential for PA promotion when used 


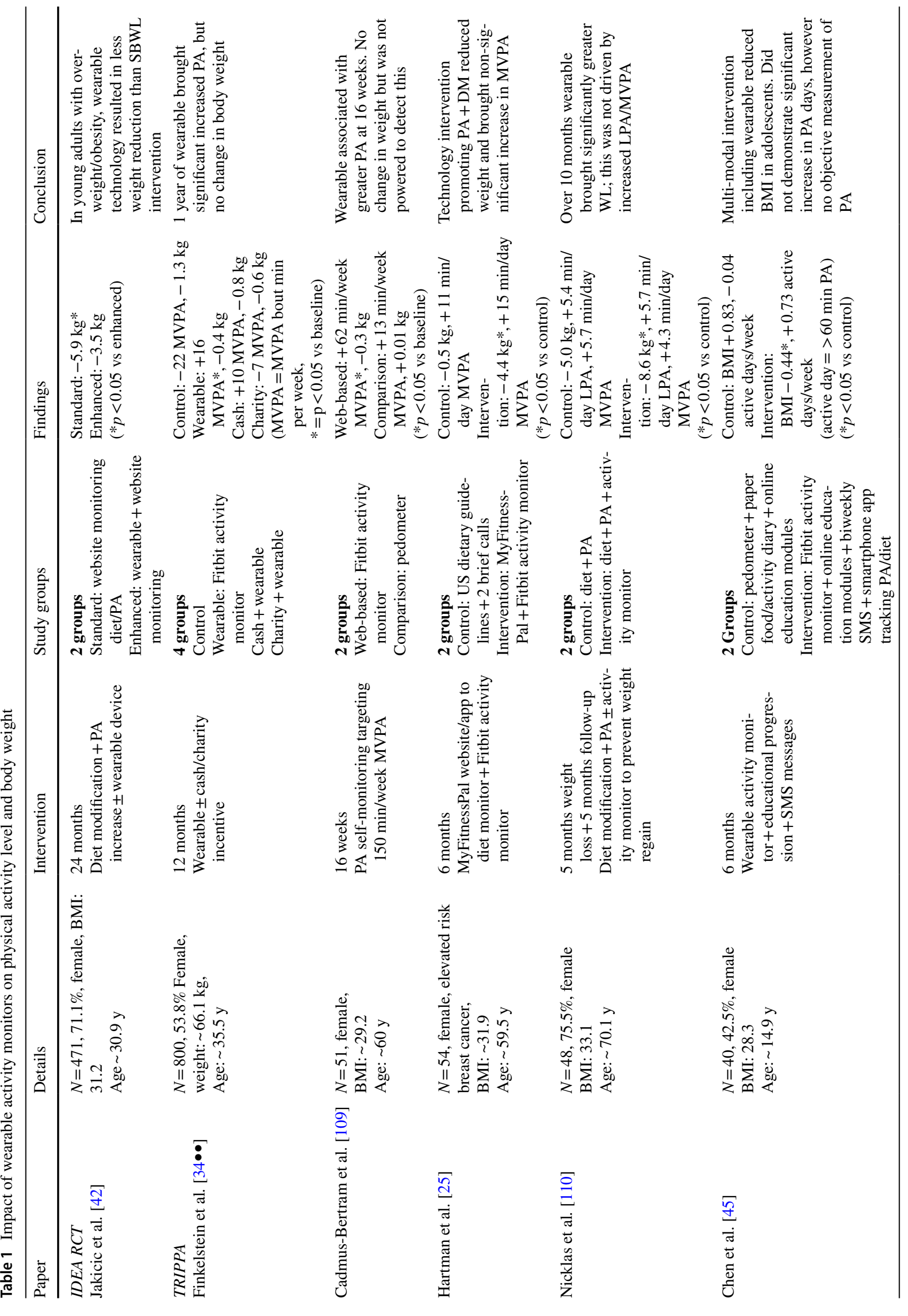




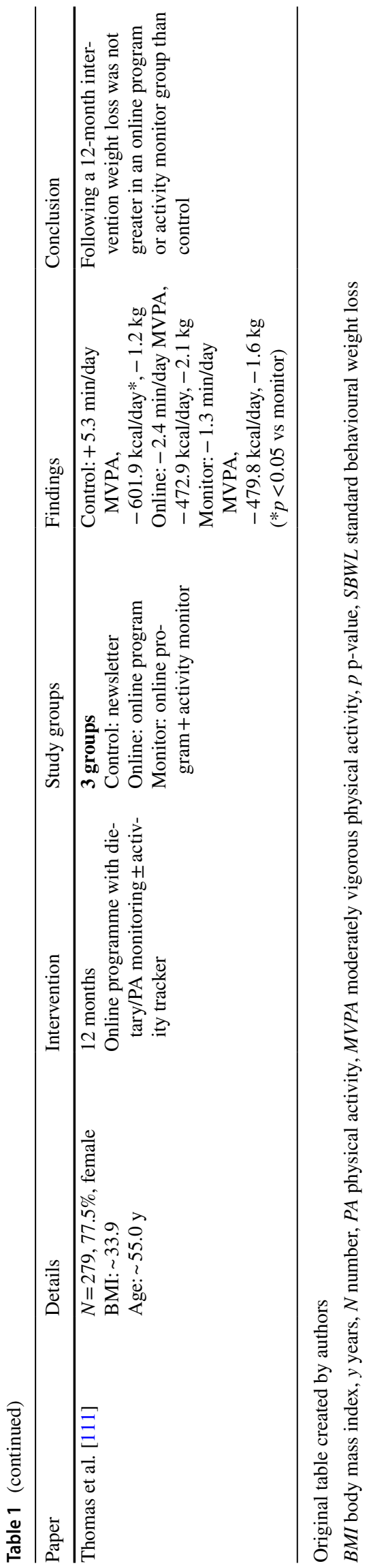

alongside standard behavioural weight loss (SBWL) programmes $[27,28]$. They also have potential to improve quality of life [29].

\section{Influence of Wearables on PA}

Wearable devices are an acceptable technology to a diverse range of populations [30-33]. The Singaporean TRIPPA study randomised 800 participants, aged 21-65 years, to four groups: a Fitbit activity tracker, tracker plus cash incentives, tracker plus charity incentive or control. Assessment of moderately vigorous physical activity (MVPA), the primary outcome measure, was made at intervention end, at 6 months and at 12 months ( 6 months post-intervention). At 6 months, the cash incentive was most effective, increasing MVPA by 29 bout $\min$ per week (95\% CI $10-47 ; p=0.0024)$. At 12 months, the activity tracker, with or without charity incentives, was effective at stemming the reduction in MVPA bout min/week observed within the control group, but there was no improvements in any health outcomes (weight or blood pressure) [34••].

Similarly, 51 inactive, overweight, post-menopausal women were randomised to activity monitor (Fitbit tracker) or standard pedometer (control). They demonstrated that activity monitoring significantly increased MVPA $(62 \pm 108 \mathrm{~min} /$ week $(p<0.01)$, and steps (by $789 \pm 1979$ $(p=0.01)$, over 16 weeks compared to non-significant increases in the control group. This pilot, however, was underpowered to detect between group differences [35].

In contrast, in Thompson et al.'s study, 49 older people (65-95 years) took part in a randomised controlled crossover study giving an accelerometer providing activity feedback with exercise counselling for 48 weeks versus no initial intervention and 24 weeks accelerometry/counselling. The eHealth intervention did not lead to significant changes in PA, body weight, percentage body fat or blood parameters $(p>0.05)[36]$. Similarly, three further RCTs ((1) 104 medical doctors, (2) 50 middle aged men, (3) 227 Americans) showed that wearables do not significantly increase the PA level [37-39]. A systematic review of studies assessing wearable activity trackers suggested that they have the potential to increase PA participation as a primary component or as part of a broader intervention, but it acknowledged often only as short-term effects. However, to be implemented within health services, they must be shown to reduce weight [15].

\section{Influence of Wearables on Body Weight}

Wearable activity monitors may enhance the effects of a SBWL intervention for weight reduction or even provide an equivalent substitute. Pellegrini et al. conducted a 3-arm intervention study in 51 people comparing an 'in-person' 
SBWL programme alone (SBWL), with a technologybased system (TECH) incorporating digital dietary and physical activity tracking capability (with weekly feedback based on these behaviours) with a combination of the two (SBWL + TECH). Body weight and physical activity were compared at baseline and after 6 months. The greatest weight loss at 6 months was seen with a combination of SBWL and TECH $(-8.8 \pm 5.0 \mathrm{~kg},-8.7 \pm 4.7 \%)$, lesser amounts with TECH alone $(-5.8 \pm 6.6 \mathrm{~kg},-6.3 \pm 7.1 \%)$ and the least with SBWL $(-3.7 \pm 5.7 \mathrm{~kg},-4.1 \pm 6.3 \%)$ ( $\mathrm{p}<0.001)$. Self-reported PA increased significantly in all groups: SBWL $(473.9 \pm 800.7 \mathrm{kcal} /$ week $)$, SBWL + TECH $(713.9 \pm 1,278.8 \mathrm{kcal} /$ week $)$ and TECH $(1,066.2 \pm 1,371 \mathrm{kcal} /$ week $)(\mathrm{p}<0.001)$. No between group differences were noted $(p=0.25)$ [40].

A larger RCT of 197 sedentary adults with overweight or obesity were randomised into 1 of 4 groups over 9 months: standard care (using a weight-loss manual), a group-based behavioural weight loss program (GWL), a SenseWear armband that tracks daily energy expenditure and energy intake (Armband) or a combination of the two (GWL + Armband). After 9 months, there was significant weight loss in all 3 intervention groups (GWL, $1.86 \mathrm{~kg}, p=0.05$; Armband alone, $3.55 \mathrm{~kg}, p=0.0002$; GWL + Armband, $6.59 \mathrm{~kg}, p<0.0001)$ but not in the control group $(0.89 \mathrm{~kg}$, $p=0.39$ ). Significant weight loss was only achieved at month 9 when comparing the GWL + Armband group to control $(p=0.04)$ [41]. These investigations highlight the supplementary effect of PA monitoring when coupled with SBWL interventions on weight.

Wearable activity monitors may have greater efficacy in middle- to older-aged populations with some study results suggesting that activity monitoring is less effective in younger populations. In the IDEA RCT study of 471 young adults, aged $18-35$ years, BMI $25-40 \mathrm{~kg} / \mathrm{m}^{2}$, a technologyenhanced weight loss intervention (including a wearable activity monitor and web interface) resulted in less weight loss than a SBWL programme (3.5 vs $5.9 \mathrm{~kg})$. However, significant improvements in body composition, fitness, PA, and diet were detected in both groups, with no significant difference between them [42]. The negative findings of the study may be confounded by wearable devices not being available from trial onset.

Similarly, the results of a smaller RCT of children with obesity, aged 10-17 years, comparing a 3-month standard weight loss intervention versus a personalised technologybased approach using a wristband to measure energy expenditure, a smartphone application to measure energy intake and weekly feedback, demonstrated equivalence [43]. However, in 48 older adults with obesity, age 65-79 years, randomised to a 5-month weight loss intervention of a hypocaloric diet, aerobic exercise with/without an accelerometer to provide real-time feedback and increase in PA/ reduce sedentary time, the addition of the feedback resulted in greater weight loss and less weight regain than that seen in the control group [44].

The use of technology to implement multi-modal interventions promoting PA and dietary modification simultaneously make it difficult to independently assess their relative contributions to the weight loss. This is exemplified in an RCT of 54 women (at increased risk of breast cancer) comparing a 6-month SBWL program with a multi-modal technology-based intervention including activity monitoring (Fitbit), a mobile app (MyFitnessPal) and phone counselling. The multi-modal intervention group lost significantly more weight, despite similar PA levels, pointing to dietary modification rather than PA as the mediating factor [25]. Similar findings were seen in Chinese American adolescents [45].

A large network meta-analysis of 31 studies conducted in individuals with overweight/obesity reported that wearable-based interventions (using accelerometer, pedometer, or commercial devices) are effective interventions for reducing body weight and body mass index [46•]. Another meta-analysis also concluded similarly, suggesting that 12 weeks/more duration of intervention is more effective and that every week of wearable use reduces weight by $0.37 \%$ [47]. Regular activity monitoring is superior to intermittent use [48]. Evidence is strongest over the short and medium term; limited long-term efficacy data is available. Furthermore, data supports their efficacy in middle- to older-aged adults; younger people have a suboptimal response, and this requires further evaluation [15]. Complex study designs, involving multiple BCTs, make it challenging to determine whether wearables can independently influence weight [27].

\section{Reproducibility of Wearables Measurements}

Multiple studies have determined the reproducibility and accuracy of wearable technologies for measurement of PA and associated energy expenditure (EE), an important consideration if these technologies are to be implemented in healthcare [13]. Accepting variable performances of different devices, step count measurement appears to be reproducible, but EE appears to be overestimated [49, 50]. Two systematic reviews also conclude that wearables provide reproducible measures of PA, but sub-optimal EE measurement $[15,51]$. Thus, it appears that PA level or step count, rather than $\mathrm{EE}$, can help participants to accurately monitor both progress and achievement of PA goals.

Overall, whilst wearable technologies can promote PA and weight loss in a healthcare setting, (Table 1); their independent effect on weight loss through increased PA is difficult to dissect from that of dietary modification. The devices show most promise in middle- to older-aged populations; younger populations may require alternative approaches, i.e. BCT. PA feedback data must be tailored to the patient group, 
ensuring it provides health behaviour insights without being overwhelmingly complex [15, 22, 23, 27, 52, 53]. Duration of use is also a consideration-most effective when used for at least 12 weeks-but longer-term efficacy is less clear $[15,47,54]$. Significant attrition does appear to be an issue. Services must ensure that the devices utilise evidence-based BCT and implement mechanisms ensuring long-term adherence given the documented $>30 \%$ attrition over 6 months and the need for sustained lifestyle changes [23].

\section{Mobile Phone Interventions (Table 2)}

Mobile phone applications (apps) have been investigated for their role in monitoring PA and influencing PA interventions. Their varied functionalities include providing PA self-management information, facilitating self-monitoring via inbuilt accelerometers and linking with external devices. They provide reproducible PA measurements but require a strong evidence base before being implemented within a healthcare setting $[55,56]$.

\section{Effect of Mobile Apps on Daily Step Count}

A variety of studies have suggested that mobile app interventions integrated with PA monitoring can increase step count [57-62]. In one large RCT of 200 people (50 intervention, 150 controls) in which a smartphone app was implemented within an existing eHealth intervention $(10,000$ steps Australia), researchers observed a greater odds of achieving $>10,000$ steps/day [63]. Similarly in short-term ( 2 weeks) studies of sedentary females $(n=42)$ and longerterm studies (6 months) of patients with T2D $(n=12)$ smartphone apps increased step count by 800 steps and 1100 steps per day respectively $[57,64]$. Overall, smartphone PA monitoring shows promise in promoting PA engagement and behaviour change when coupled with other PA interventions.

\section{Effects of Mobile Apps on Weight Loss}

Smartphone apps integrated with pedometers are linked with weight loss $[62,65,66]$. In 61 patients with obesity at high risk of T2D, a standard intervention, based on the Diabetes Prevention Program, was compared with an intervention comprising reduced in person contact substituted with a combined mobile app and pedometer intervention to facilitate self-monitoring. Smartphone self-monitoring $(n=30)$ was associated with greater weight loss than the control intervention (weight change: $-6.2 \mathrm{vs}+0.3 \mathrm{~kg}$ ) and higher PA levels (+2551 vs -734 steps/day) [58].

Similar to the phenomenon observed with wearables, smartphone app/mobile interventions have a less pronounced effect in younger populations. In a 35-day intervention (children/adolescents), smartphone monitoring increased PA and reduced weight $[61,67]$. Yet, in a large RCT of 365 young adults with overweight/obesity, a smartphone self-monitoring app, which utilised evidence-based BCTs, did not change PA or body weight [68]. Likewise in the ATLAS-RCT in adolescent males, a 20 -week multi-modal intervention, including smartphone self-monitoring, did not bring greater weight loss or PA levels [69]. The efficacy of mobile phone apps is summarised by a meta-analysis $(n=12$ studies) reporting a -1 kg body weight reduction but no significant difference in PA level [70].

Mobile apps have been examined against paper diary monitoring with somewhat conflicting results. In a posthoc analysis of a 6-month RCT of 96 overweight men and women, 6 months mobile app self-monitoring was reported to increase exercise self-monitoring and PA and reduce BMI to a greater extent than non-app users. [71] Carter et al. reported the greatest mean weight change in 128 overweight volunteers at 6 months with a smartphone app compared with a diary and a website group [72]. Yet, a separate RCT of mobile app PA and diet monitoring highlighted that whilst the intervention caused weight loss, PA level actually reduced [73]. Likewise, a primary care RCT $(n=212)$ interrogating the smartphone self-monitoring app MyFitnessPal reduced weight but did not alter PA level with significant decline in use after 1 month noted [74].

Even though dietary intake and PA are monitored concurrently in all studies, evaluation of their relative impacts on weight loss is challenging; self-monitoring mobile apps likely predominantly affect behaviour change through dietary modification rather than increased PA. Akin to the data in wearables, supporting data for apps is strongest in the short to medium term, with limited evidence interrogating long-term efficacy $[70,75]$. Unlike wearables, evidencebased BCTs are not commonly utilised by mobile phone apps which may partly explain their heterogeneous effects on PA [76].

\section{Short Message Service (Table 3)}

Short Message Service (SMS) has been utilised as an eHealth intervention whereby individuals receive text messages encouraging behaviour changes including PA [77, 78]. In a 12-month cluster RCT of 250 women, SMS reinforcement utilised alongside a SBWL program resulted in a small difference in weight change between the intervention and control groups at 12 months $(-1.1 \mathrm{~kg})$ with beneficial changes in PA and diet [79]. In an RCT of 52 college students, an 8-week multi-modal intervention including Facebook and Facebook plus SMS demonstrated that the addition of SMS behavioural advice achieved significant weight loss $(\sim 2.4 \mathrm{~kg})$ [80]. Yet, when implemented in 170 individuals with obesity 


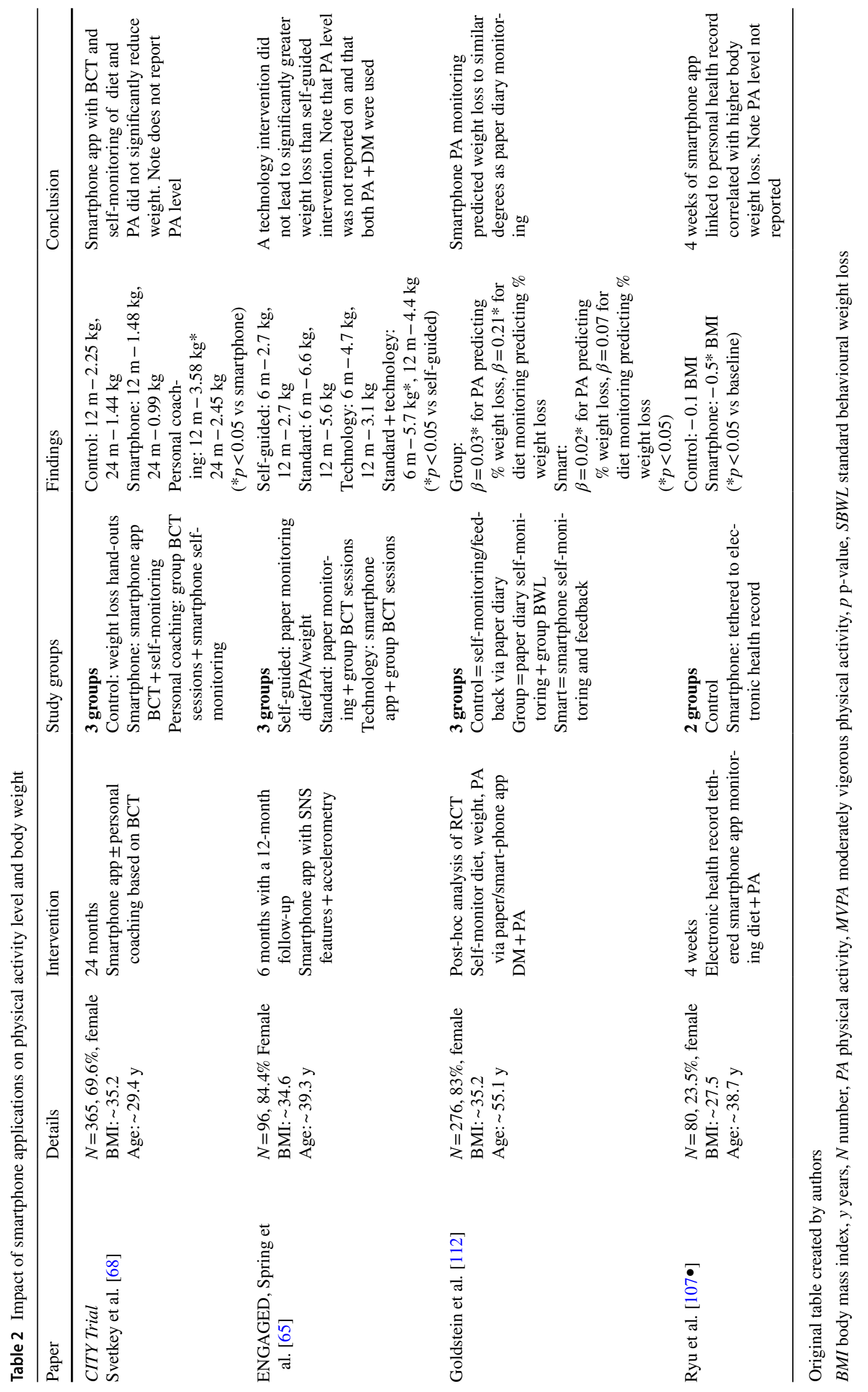




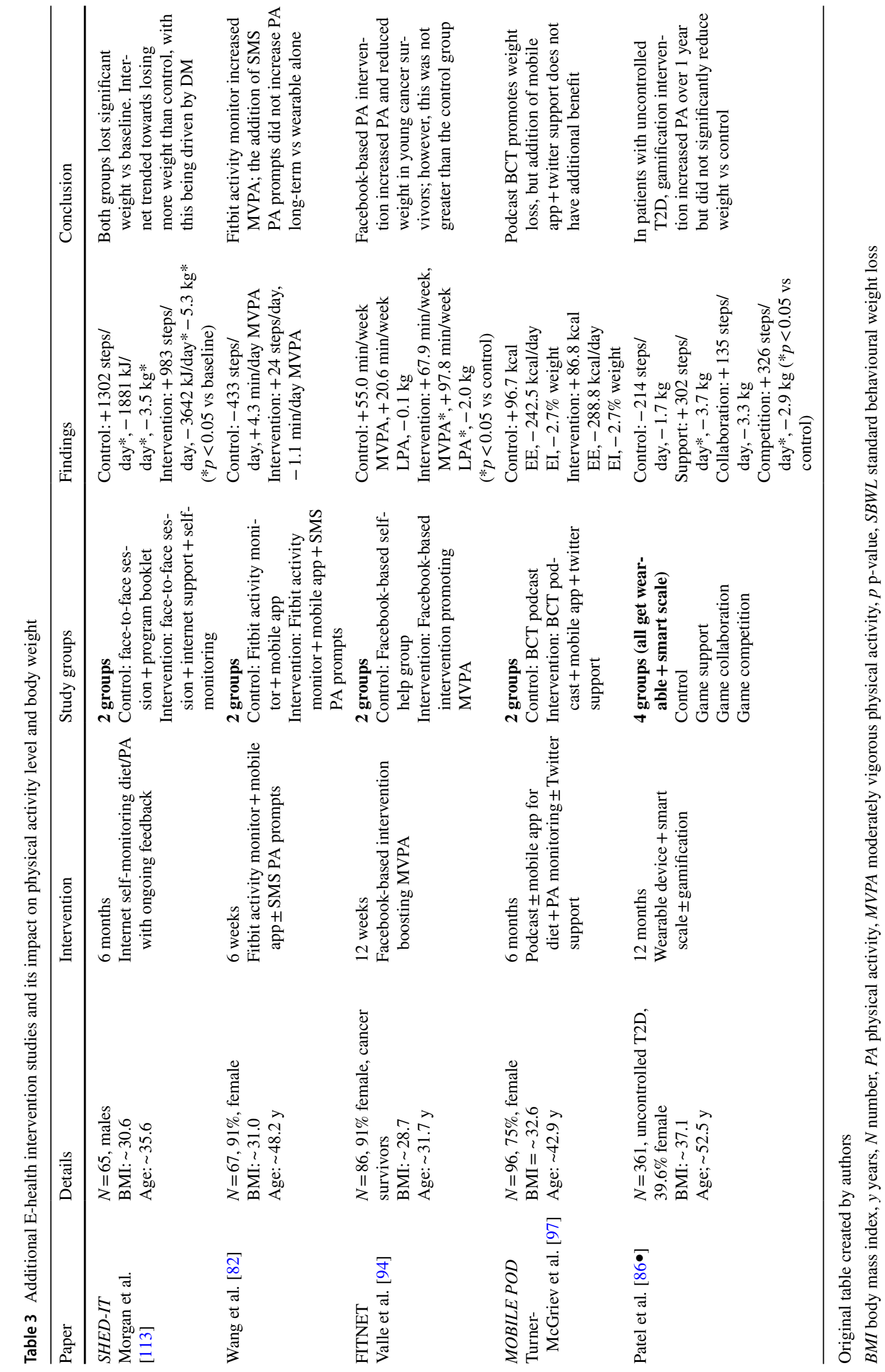


daily SMS incorporating PA feedback increased step count by 3000 steps/day but did not lead to weight loss. This trial did report significant attrition potentially confounding find-ings [81]. Similarly, Wang et al. demonstrated the combina-tion of SMS exercise prompts and wearables did not increase PA more than wearables alone [82].

Overall, a meta-analysis including only 6 studies showed that the weighted mean change in body weight with SMS intervention was $-2.56 \mathrm{~kg}$, although importantly both PA and dietary modification were concurrently evaluated and there was a lack of long-term data [83]. Future studies should assess the impact of incorporating BCTs by utilising accelerometry-determined PA data to provide personalised SMS. Whether SMS PA interventions are particularly relevant to older individuals or those from countries where smartphones are less readily available is also unknown. More research is required before SMS PA interventions can be implemented within a healthcare environment.

\section{Exergaming (Table 3)}

Exergaming is a videogame requiring body movements and is available across many systems including traditional consoles and smartphones. It is an innovative intervention which has considerable potential to promote PA across all age groups and in particular younger demographics [8, $84,85,86 \bullet$. Using accelerometry, it has been shown that when children exergame they are physically active $50 \%$ of the time, with $20 \%$ of time in moderate-vigorous physical activity and 30\% in light PA [85]. Indeed, one 2-year intervention study of 261 school children demonstrated that exergaming proved equivalent to in-person PE classes in stimulating accelerometry-defined PA [87].

The technology has even shown promise in adult populations, with 10 weeks of combined exergaming and activity monitoring increasing PA more than activity monitoring alone [88]. In a 12-week RCT of 40 participants, a mobile phone exergame was shown to bring higher PA levels relative to control with no difference in body weight reported [89]. In addition, college students partaking in regular exercise expend greater energy than when exergaming [90]. In contast, 12 weeks of exergaming in 37 overweight girls did not significantly improve accelerometry defined PA [91].

Overall, the evidence indicates that whilst exergaming has potential to improve PA, there is a paucity of data evaluating exergaming as a weight loss strategy [84]. Future studies need to investigate the long-term efficacy of exergaming for PA promotion and weight reduction. A key question to answer is whether exergaming replaces sedentary screen time or displaces an individual's usual sports/PA. If the latter, then exergaming would be best promoted in the most sedentary individuals [13].

\section{Social Networking Sites (Table 3)}

Social networking sites (SNS) have been interrogated for their utility in promoting PA and weight loss [92-94]. An example of this is Facebook or the sports SNS Strava, which allows exercisers to document their PA and monitor other users/friends' progress. SNS provide a unique social interface whereby individuals can be influenced by their network's positive health behaviours without direct contact [92, 95-97]. The effects are mediated at least partly by the power of social influence [95].

In the FITNET study of 86 cancer survivors, a 12-week SNS PA intervention increased light PA and brought greater weight loss than control $(-2.0 \mathrm{~kg})$ [94]. Whilst in the ManUp RCT of 301 middle aged male participants, an IT-based intervention (Web and Mobile App) including SNS support was as effective in improving PA and dietary behaviours as print-based methods [96]. Similarly, Pope et al. reported that an SNS intervention increased PA to similar degrees as a wearable activity monitor. Importantly, participants found the wearable challenging to use potentially biasing results [98, 99].

Overall, whilst SNS interventions may be a useful PA stimulus, data is based on multi-modal interventions making evaluation of their effect on PA and body weight impossible. Most studies have focused on Facebook; future studies should target more recently developed SNS platforms including Instagram, TikTok, WhatsApp and Snapchat. These SNSs generally appeal to differing demographics than Facebook and may prove more successful. Studies must also isolate how to maximise engagement in SNS health interventions and how the intervention could be utilised in the healthcare system $[8,100,101]$. Table 3 provides further studies evaluating SNS, exergaming and SMS interventions.

\section{Internet-Based Interventions (Table 3)}

A variety of internet-based interventions have been evaluated for their ability to promote PA and weight loss including emails, website self-monitoring, smart-scales and podcasts [102-104]. In a 12-week RCT, a weight loss podcast providing diet and PA advice demonstrated significant efficacy in improving MVPA and weight versus control [104]. In the SHED-IT RCT of 65 male University staff and students with overweight, an Internet-based weight-loss program with PA monitoring brought significantly more weight loss at 6 months vs control $(-5.3 \mathrm{~kg}$ vs $-3.5 \mathrm{~kg})$. The weight loss was driven by dietary modification, with no change in PA noted [103]. A common theme in many of these studies is that whilst interventions like smart-scales, 
email counselling and website self-monitoring might promote weight loss, this seems to be achieved predominantly via dietary modification with weak evidence to support internet-based interventions achieving weight loss independently by increasing PA.

\section{Future Directions}

\section{Design of Future eHealth Intervention Studies}

There is considerable scope to design robust eHealth intervention trials to inform the development of a strong evidence base and facilitate integration of eHealth technologies into healthcare. Factors to consider include (i) the independent interrogation of interventions (dietary vs PA), (ii) a focus on body fat distribution rather than simple weight change, (iii) demonstration of long-term efficacy and of weight loss maintenance, (iv) assessment of emerging eHealth interventions and (v) provision of real-world data in a healthcare service.

Factorial trial designs will facilitate quantification of the individual effect of eHealth interventions on PA and body weight. Given that data has demonstrated eHealth interventions reduce central obesity, research should assess the impact of eHealth interventions on ectopic fat depots in the liver, skeletal muscle and heart, given their pathophysiological link with T2D, non-alcoholic fatty liver disease (NAFLD) and cardiovascular disease (CVD) [105]. Longterm data will allow determination of whether PA behaviour changes are sustained beyond 12 months with a dearth of evidence for their efficacy beyond this time point. Maintenance of weight loss is currently a field where eHealth interventions have not been rigorously evaluated; yet, weight regain is common problem so novel approaches are required $[16,106]$. Future trials should also interrogate the efficacy of emerging internet-based PA platforms that provide workouts linked to wearable devices and include Apple Fitness, Fiit and programmes provided via YouTube. Health services must also ensure that the populations at greatest need, such as the socio-economically deprived and older individuals (who have greater risk of obesity), have access to eHealth interventions. Finally, trials should focus on creating realworld data of eHealth interventions within health services. Ryu et al. showed that an eHealth intervention linked to electronic patient records was effective in the short term [107•]. Long-term data must be analysed in a similar intervention to demonstrate efficacy. This development will allow closer monitoring of patient progress, highlighting early attrition and consequently facilitating more intensive therapies when necessitated.

\section{Potential Developments in eHealth Interventions}

There is considerable scope for future eHealth innovations considering evolution of artificial intelligence. For example, data-driven feedback from wearables and smartphones may encourage attainment of personalised PA goals via digital personal assistants like Apple Siri or Google Now and digital health coaching (using evidence-based BCTs). Furthermore, there is potential to remotely monitor heart rate using wearable devices to guide physical activity goals, providing real-time feedback to individuals regarding time in MVPA. Additionally, behavioural economics could be incorporated into developing programmes whereby participants are rewarded for increased PA. A lottery could be implemented, whereby individuals are motivated to maintain a behaviour
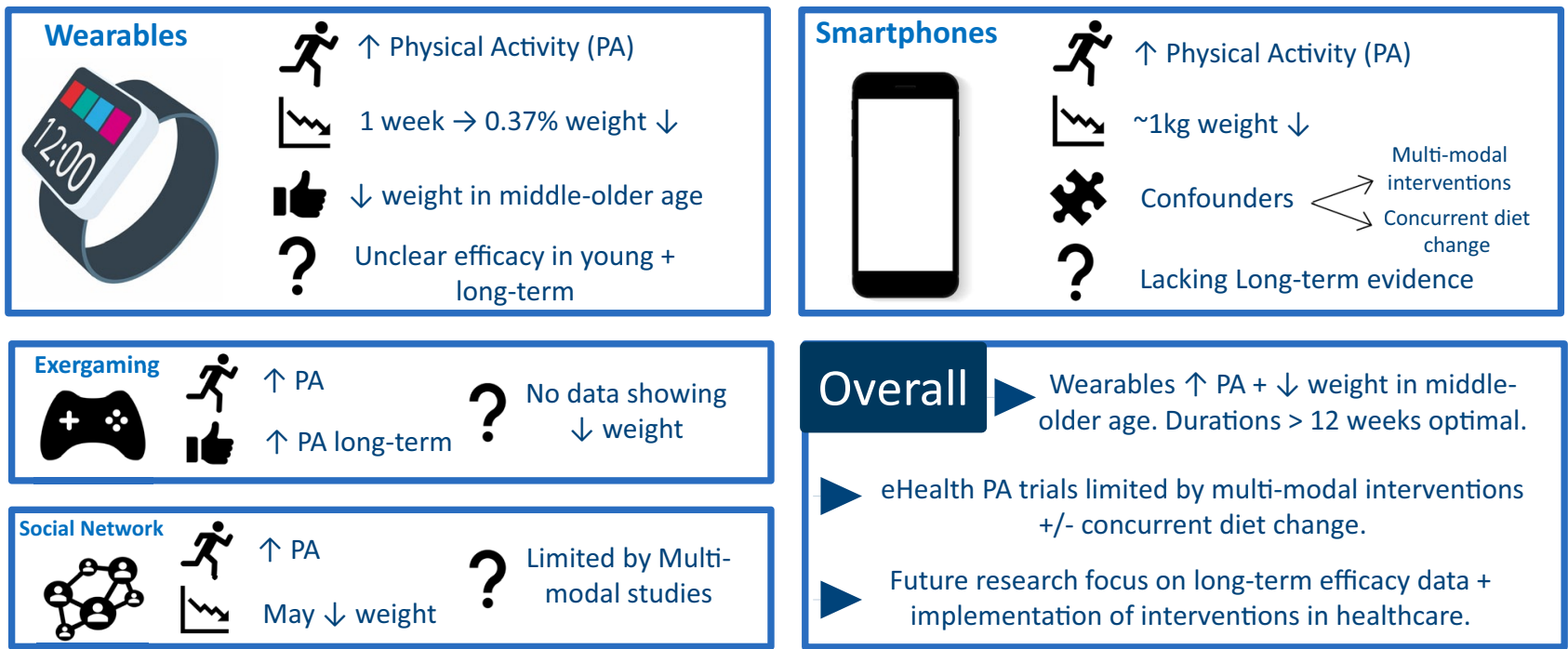

Fig. 1 Graphical summary of review findings. Original figure created by authors. PA=physical activity 
due to the chance of winning. This has potential to reduce attrition and improve adherence to eHealth interventions [23, 108].

\section{Conclusions (Fig. 1)}

Overall eHealth interventions provide a novel approach to promote PA and weight reduction. Figure 1 provides a graphical summary of the review's findings. Wearable technologies may increase PA and promote weight loss in middle to older aged adults with durations of $>12$ weeks being optimal, although the duration of effect is uncertain, with little/ no evidence beyond 12 months. Smartphone interventions promote weight loss, but due to complex trial designs it is unclear whether this is driven by PA or dietary modification. Exergaming, SNS programmes and SMS reduce weight in some cases, yet there is a lack of long-term data and it is unclear whether PA modification is the mechanistic driver or rather dietary change. Altogether, much more research is needed, particularly with longer-term efficacy data, to demonstrate significant and convincing effects on physical activity patterns or on body weight before eHealth interventions can be implemented within healthcare services. However, it would appear clear that technology will become an asset for health care in the twenty-first century and opportunities exist to make best use of it.

Author Contribution The initial literature review was performed by LJD and DJC. The first draft of the manuscript was written by LJD and DJC with all authors commenting on subsequent versions of the manuscript. All authors read and approved the final manuscript.

\section{Declarations}

Conflict of Interest AAT reports grants, personal fees, and travel support from Sanofi; grants, personal fees and educational events grants from Novo Nordisk; travel support from Merck Sharp and Dohme; personal fees and travel support from Boehringer Ingelheim; personal fees from Lilly, AstraZeneca, Bristol-Myers Squibb, and Janssen; equipment and travel support from ResMed; equipment from Philips Respironics, Impeto Medical, and ANSAR Medical Technologies; grants and non-financial support from Napp; and equipment and support staff from BHR Pharmaceuticals Ltd. AAT is currently an employee of Novo Nordisk. This work was performed before AAT becoming a Novo Nordisk employee, and Novo Nordisk had no role in this study.

Human and Animal Rights and Informed Consent This article does not contain any studies with human or animal subjects performed by any of the authors.

Open Access This article is licensed under a Creative Commons Attribution 4.0 International License, which permits use, sharing, adaptation, distribution and reproduction in any medium or format, as long as you give appropriate credit to the original author(s) and the source, provide a link to the Creative Commons licence, and indicate if changes were made. The images or other third party material in this article are included in the article's Creative Commons licence, unless indicated otherwise in a credit line to the material. If material is not included in the article's Creative Commons licence and your intended use is not permitted by statutory regulation or exceeds the permitted use, you will need to obtain permission directly from the copyright holder. To view a copy of this licence, visit http://creativecommons. org/licenses/by/4.0/.

\section{References}

Papers of particular interest, published recently, have been highlighted as:

- Of importance

$\bullet$ Of major importance

1. Guthold R, Stevens GA, Riley LM, Bull FC. Worldwide trends in insufficient physical activity from 2001 to 2016: a pooled analysis of 358 population-based surveys with 1.9 million participants. Lancet Glob Health. 2018;6:e1077-86.

2. Global Physical Activity Action Plan 2018-2030.

3. Bull FC, Al-Ansari SS, Biddle S, et al. World Health Organization 2020 guidelines on physical activity and sedentary behaviour. Br J Sports Med. 2020;54:1451-62.

4. Kissler HJ, Settmacher U. Bariatric surgery to treat obesity. Semin Nephrol. 2013;33:75-89.

5. Shaw K, Gennat H, O'Rourke P, Del Mar C. Exercise for overweight or obesity. Cochrane Database Syst Rev. 2006:CD003817.

6. Victoria AC. The role of physical activity in producing and maintaining weight loss. Nat Clin Pract Endocrinol Metab. 2007;176:139-48.

7. Foster-Schubert KE, Alfano CM, Duggan CR, et al. Effect of diet and exercise, alone or combined, on weight and body composition in overweight-to-obese postmenopausal women. Obesity. 2012;20:1628-38.

8. Gao Z, Lee JE. Emerging technology in promoting physical activity and health: challenges and opportunities. J Clin Med. 2019;8.

9. Carbone S, Del Buono MG, Ozemek C, Lavie CJ. Obesity, risk of diabetes and role of physical activity, exercise training and cardiorespiratory fitness. Prog Cardiovasc Dis. 2019;62:327-33.

10. Morris JNHJ, Raffle PA, Roberts CG, Parks JW. Coronary heartdisease and physical activity of work. Lancet. 1953;262:1053-7.

11. Chin SH, Kahathuduwa CN, Binks M. Physical activity and obesity: what we know and what we need to know. Obes Rev. 2016; 17:1226-44.

12. Wen CP, Wai JPM, Tsai MK, et al. Minimum amount of physical activity for reduced mortality and extended life expectancy: a prospective cohort study. The Lancet. 2011;378:1244-53.

13. Gao L. Emerging technology in promoting physical activity and health: challenges and opportunities. J Clin Med. 2019;8:1830.

14. Gordon PM, Heath GW, Holmes A, Christy D. The quantity and quality of physical activity among those trying to lose weight. Am J Prev Med. 2000;18:83-6.

15. Cheatham SW, Stull KR, Fantigrassi M, Motel I. The efficacy of wearable activity tracking technology as part of a weight loss program: a systematic review. J Sports Med Phys Fitness. 2018;58:534-48.

16. Hutchesson MJ, Rollo ME, Krukowski R, et al. eHealth interventions for the prevention and treatment of overweight and obesity in adults: a systematic review with meta-analysis. Obes Rev. 2015; 16:376-92. 
17. Patel ML, Wakayama LN, Bennett GG. Self-monitoring via digital health in weight loss interventions: a systematic review among adults with overweight or obesity. Obesity (Silver Spring). 2021;29:478-99.

18. Michie S, Richardson M, Johnston M, et al. The behavior change technique taxonomy (v1) of 93 hierarchically clustered techniques: building an international consensus for the reporting of behavior change interventions. Ann Behav Med. 2013;46:81-95.

19. Carels RA, Darby LA, Rydin S, et al. The relationship between self-monitoring, outcome expectancies, difficulties with eating and exercise, and physical activity and weight loss treatment outcomes. Ann Behav Med. 2005;30:182-90.

20. Helsel DL, Jakicic JM, Otto AD. Comparison of techniques for self-monitoring eating and exercise behaviors on weight loss in a correspondence-based intervention. J Am Diet Assoc. 2007; 107:1807-10.

21. Burke LE, Wang J, Sevick MA. Self-monitoring in weight loss: a systematic review of the literature. J Am Diet Assoc. 2011;111:92-102.

22. Piwek L, Ellis DA, Andrews S, Joinson A. The rise of consumer health wearables: promises and barriers. PLoS Med. 2016;13:e1001953.

23. Patel MS, Asch DA, Volpp KG. Wearable devices as facilitators, not drivers, of health behavior change. JAMA. 2015;313:459-60.

24. Fawcett E, Van Velthoven MH, Meinert E. Long-term weight management using wearable technology in overweight and obese adults: systematic review. JMIR Mhealth Uhealth. 2020;8:e13461.

25. Hartman SJ, Nelson SH, Cadmus-Bertram LA, et al. Technologyand phone-based weight loss intervention: pilot RCT in women at elevated breast cancer risk. Am J Prev Med. 2016;51:714-21.

26. Butryn MLPS, Hill JO, Wing RR. Consistent self-monitoring of weight: a key component of successful weight loss maintenance. Obesity. 2007;15:3091-6.

27. Riffenburg KM, Spartano NL. Physical activity and weight maintenance: the utility of wearable devices and mobile health technology in research and clinical settings. Curr Opin Endocrinol Diabetes Obes. 2018;25:310-4.

28. Martin CK, Miller AC, Thomas DM, et al. Efficacy of SmartLoss, a smartphone-based weight loss intervention: results from a randomized controlled trial. Obesity (Silver Spring). 2015;23:935-42.

29. Effect of Fitbit and iPad wearable technology in health-related quality of life in adolescent and young adult cancer patients. $\mathbf{J}$ Adolescent and Young Adult Oncol. 2018;7:579-583.

30. Karapanos E, Gouveia R, Hassenzahl M, Forlizzi J. Wellbeing in the making: peoples' experiences with wearable activity trackers. Psychol Well Being. 2016;6:4.

31. Bentley CL, Otesile O, Bacigalupo R, et al. Feasibility study of portable technology for weight loss and $\mathrm{HbAlc}$ control in type 2 diabetes. BMC Med Inform Decis Mak. 2016;16:92.

32. Lyons EJ, Swartz MC, Lewis ZH, et al. Feasibility and acceptability of a wearable technology physical activity intervention with telephone counseling for mid-aged and older adults: a randomized controlled pilot trial. JMIR Mhealth Uhealth. 2017;5:e28.

33. Mercer K, Giangregorio L, Schneider E, et al. Acceptance of commercially available wearable activity trackers among adults aged over 50 and with chronic illness: a mixed-methods evaluation. JMIR Mhealth Uhealth. 2016;4:e7.

34.• Finkelstein EA, Haaland BA, Bilger M, et al. Effectiveness of activity trackers with and without incentives to increase physical activity (TRIPPA): a randomised controlled trial. Lancet Diabetes Endocrinol. 2016;4:983-95. (One year of wearable activity monitors brought significant increase in physical activity, but no change in body weight.)
35. Cadmus-Bertram LA, Marcus BH, Patterson RE, et al. Randomized trial of a Fitbit-based physical activity intervention for women. Am J Prev Med. 2015;49:414-8.

36. Thompson WG, Kuhle CL, Koepp GA, et al. "Go4Life" exercise counseling, accelerometer feedback, and activity levels in older people. Arch Gerontol Geriatr. 2014;58:314-9.

37. Thorndike AN, Mills S, Sonnenberg L et al. Activity monitor intervention to promote physical activity of physicians-in-training: randomized controlled trial. PLoS One. 2014;9:e100251.

38. Ashton LM, Morgan PJ, Hutchesson MJ, et al. Feasibility and preliminary efficacy of the "HEYMAN" healthy lifestyle program for young men: a pilot randomised controlled trial. Nutr J. 2017;16:2.

39. Van Hoye K, Boen F, Lefevre J. The impact of different degrees of feedback on physical activity levels: a 4-week intervention study. Int J Environ Res Public Health. 2015;12:6561-81.

40. Pellegrini CA, Verba SD, Otto AD, et al. The comparison of a technology-based system and an in-person behavioral weight loss intervention. Obesity. 2012;20:356-63.

41. Shuger SL, Barry VW, Sui X, et al. Electronic feedback in a diet- and physical activity-based lifestyle intervention for weight loss: a randomized controlled trial. Int J Behav Nutr Phys Act. 2011;8:41.

42. Jakicic JM, Davis KK, Rogers RJ, et al. Effect of wearable technology combined with a lifestyle intervention on longterm weight loss: the IDEA randomized clinical trial. JAMA. 2016;316:1161-71.

43. Mameli C, Brunetti D, Colombo V, et al. Combined use of a wristband and a smartphone to reduce body weight in obese children: randomized controlled trial. Pediatr Obes. 2018;13:81-7.

44. Nicklas BJ, Gaukstern JE, Beavers KM, et al. Self-monitoring of spontaneous physical activity and sedentary behavior to prevent weight regain in older adults. Obesity. 2014;22:1406-12.

45. Chen JL, Guedes CM, Lung AE. Smartphone-based healthy weight management intervention for Chinese American adolescents: short-term efficacy and factors associated with decreased weight. J Adolesc Health. 2019;64:443-9.

46. McDonough DJ, Su X, Gao Z. Health wearable devices for weight and BMI reduction in individuals with overweight/obesity and chronic comorbidities: systematic review and network meta-analysis. British Journal of Sports Medicine 2021:bjsports-2020-103594. Health wearable devices are effective interventions for reducing body weight in individuals with overweight/obesity. Interventions of at least 12 weeks in duration are most effective for achieving weight loss.

47. Yen H-Y, Chiu H-L. The effectiveness of wearable technologies as physical activity interventions in weight control: a systematic review and meta-analysis of randomized controlled trials. Obes Rev. 2019;20:1485-93.

48. Polzien KM, Jakicic JM, Tate DF, Otto AD. The efficacy of a technology-based system in a short-term behavioral weight loss intervention. Obesity (Silver Spring). 2007;15:825-30.

49. Kaewkannate K, Kim S. A comparison of wearable fitness devices. BMC Public Health. 2016;16:433.

50. Falter M, Budts W, Goetschalckx K, et al. Accuracy of Apple Watch measurements for heart rate and energy expenditure in patients with cardiovascular disease: Cross-Sectional Study. JMIR Mhealth Uhealth. 2019;7:e11889.

51. Evenson KR, Goto MM, Furberg RD. Systematic review of the validity and reliability of consumer-wearable activity trackers. Int J Behav Nutr Phys Act. 2015;12:159.

52. Mercer K, Li M, Giangregorio L, et al. Behavior change techniques present in wearable activity trackers: a critical analysis. JMIR Mhealth Uhealth, 2016;4:e40. 
53. The Lancet Digital H. Wearable technology and lifestyle management: the fight against obesity and diabetes. The Lancet Digital Health. 2019;1.

54. McDonough DJ, Su X, Gao Z. Health wearable devices for weight and BMI reduction in individuals with overweight/obesity and chronic comorbidities: systematic review and network meta-analysis. Br J Sports Med. 2021.

55. Johnson JL, Slentz CA, Houmard JA, et al. Exercise training amount and intensity effects on metabolic syndrome (from studies of a targeted risk reduction intervention through defined exercise). Am J Cardiol. 2007;100:1759-66.

56. Bort-Roig J, Gilson ND, Puig-Ribera A, et al. Measuring and influencing physical activity with smartphone technology: a systematic review. Sports Med. 2014;44:671-86.

57. Arsand E, Tatara N, Ostengen G, Hartvigsen G. Mobile phonebased self-management tools for type 2 diabetes: the few touch application. J Diabetes Sci Technol. 2010;4:328-36.

58. Fukuoka Y, Gay CL, Joiner KL, Vittinghoff E. A novel diabetes prevention intervention using a mobile app: a randomized controlled trial with overweight adults at risk. Am J Prev Med. 2015;49:223-37.

59. Glynn LG, Hayes PS, Casey M, et al. Effectiveness of a smartphone application to promote physical activity in primary care: the SMART MOVE randomised controlled trial. Br J Gen Pract. 2014;64:e384-391.

60. Kirwan M, Duncan MJ, Vandelanotte C, Mummery WK. Using smartphone technology to monitor physical activity in the 10,000 Steps program: a matched case-control trial. J Med Internet Res. 2012;14:e55.

61. Schiel R, Kaps A, Bieber G, et al. Identification of determinants for weight reduction in overweight and obese children and adolescents. J Telemed Telecare. 2010;16:368-73.

62. Plotnikoff RC, Wilczynska M, Cohen KE, et al. Integrating smartphone technology, social support and the outdoor physical environment to improve fitness among adults at risk of, or diagnosed with, Type 2 Diabetes: Findings from the "eCoFit" randomized controlled trial. Prev Med. 2017;105:404-11.

63. Kirwan M, Duncan MJ, Vandelanotte C, Mummery WK. Using smartphone Techology to Monitor Physical Activity in the 10,000 Steps Program: A Matched Case-Control Trial. J Med Internet Res. 2012;14(2):1-10.

64. Fukuoka Y, Vittinghoff E, Jong SS, Haskell W. Innovation to motivation-pilot study of a mobile phone intervention to increase physical activity among sedentary women. Prev Med. 2010;51:287-9.

65. Spring B, Pellegrini CA, Pfammatter A, et al. Effects of an abbreviated obesity intervention supported by mobile technology: the ENGAGED randomized clinical trial. Obesity (Silver Spring). 2017;25:1191-8.

66. Ross KM, Wing RR. Impact of newer self-monitoring technology and brief phone-based intervention on weight loss: a randomized pilot study. Obesity (Silver Spring). 2016;24:1653-9.

67. Schiel R, Thomas A, Kaps A, Bieber G. An innovative telemedical support system to measure physical activity in children and adolescents with type 1 diabetes mellitus. Exp Clin Endocrinol Diabetes. 2011;119:565-8.

68. Svetkey LP, Batch BC, Lin PH, et al. Cell phone intervention for you (CITY): A randomized, controlled trial of behavioral weight loss intervention for young adults using mobile technology. Obesity (Silver Spring). 2015;23:2133-41.

69. Smith JJ, Morgan PJ, Plotnikoff RC, et al. Smart-phone obesity prevention trial for adolescent boys in low-income communities: the ATLAS RCT. Pediatrics. 2014;134:e723-731.

70. Flores Mateo G, Granado-Font E, Ferre-Grau C, MontanaCarreras X. Mobile phone apps to promote weight loss and increase physical activity: a systematic review and metaanalysis. J Med Internet Res. 2015;17:e253.

71. Turner-McGrievy GM, Beets MW, Moore JB, et al. Comparison of traditional versus mobile app self-monitoring of physical activity and dietary intake among overweight adults participating in an mHealth weight loss program. J Am Med Inform Assoc. 2013;20:513-8.

72. Carter MC, Burley VJ, Nykjaer C, Cade JE. Adherence to a smartphone application for weight loss compared to website and paper diary: pilot randomized controlled trial. J Med Internet Res. 2013;15:e32.

73. Allen JK, Stephens J, Dennison Himmelfarb CR et al. Randomized controlled pilot study testing use of smartphone technology for obesity treatment. J Obes. 2013;2013:151597.

74. Laing BY, Mangione CM, Tseng CH, et al. Effectiveness of a smartphone application for weight loss compared with usual care in overweight primary care patients: a randomized, controlled trial. Ann Intern Med. 2014;161:S5-12.

75. Bacigalupo R, Cudd P, Littlewood C, et al. Interventions employing mobile technology for overweight and obesity: an early systematic review of randomized controlled trials. Obes Rev. 2013;14:279-91.

76. Conroy DE, Yang CH, Maher JP. Behavior change techniques in top-ranked mobile apps for physical activity. Am J Prev Med. 2014;46:649-52.

77. Yoo HJ, Park MS, Kim TN, et al. A Ubiquitous Chronic Disease Care system using cellular phones and the internet. Diabet Med. 2009;26:628-35.

78. Shaw R, Bosworth H. Short message service (SMS) text messaging as an intervention medium for weight loss: a literature review. Health Informatics J. 2012;18:235-50.

79. Lombard C, Deeks A, Jolley D, et al. A low intensity, community based lifestyle programme to prevent weight gain in women with young children: cluster randomised controlled trial. BMJ. 2010;341:c3215.

80. Napolitano MA, Hayes S, Bennett GG, et al. Using Facebook and text messaging to deliver a weight loss program to college students. Obesity. 2013;21:25-31.

81. Shapiro JR, Koro T, Doran N, et al. Text4Diet: a randomized controlled study using text messaging for weight loss behaviors. Prev Med. 2012;55:412-7.

82. Wang JB, Cadmus-Bertram LA, Natarajan L, et al. Wearable sensor/device (Fitbit One) and SMS text-messaging prompts to increase physical activity in overweight and obese adults: a randomized controlled trial. Telemed J E Health. 2015;21:782-92.

83. Siopis G, Chey T, Allman-Farinelli M. A systematic review and meta-analysis of interventions for weight management using text messaging. J Hum Nutr Diet. 2015;28(Suppl 2):1-15.

84. Benzing V, Schmidt M. Exergaming for children and adolescents: strengths, weaknesses, opportunities and threats. J Clin Med. 2018;7.

85. Quan M, Pope Z, Gao Z. Examining young children's physical activity and sedentary behaviors in an exergaming program using accelerometry. J Clin Med 2018; 7.

86. Patel MS, Small DS, Harrison JD, et al. Effect of behaviorally designed gamification with social incentives on lifestyle modification among adults with uncontrolled diabetes: a randomized clinical trial. JAMA Netw Open. 2021; 4:e2110255. In patients with uncontrolled type 2 diabetes, a gamification intervention increased PA over 1 year but did not significantly reduce weight

87. Gao Z, Pope Z, Lee JE, et al. Impact of exergaming on young children's school day energy expenditure and moderate-to-vigorous physical activity levels. J Sport Health Sci. 2017;6:11-6.

88. Gremaud AL, Carr LJ, Simmering JE, et al. Gamifying accelerometer use increases physical activity levels of sedentary office workers. J Am Heart Assoc. 2018;7. 
89. Cowdery J, Majeske P, Frank R, Brown D. Exergame apps and physical activity: the results of the ZOMBIE Trial. Am J Health Educ. 2015;46:216-22.

90. McDonough DJ, Pope ZC, Zeng N, et al. Comparison of college students' energy expenditure, physical activity, and enjoyment during exergaming and traditional exercise. J Clin Med. 2018;7.

91. Staiano AE, Beyl RA, Hsia DS, et al. Twelve weeks of dance exergaming in overweight and obese adolescent girls: transfer effects on physical activity, screen time, and self-efficacy. J Sport Health Sci. 2017;6:4-10.

92. Cavallo DN, Tate DF, Ries AV, et al. A social media-based physical activity intervention: a randomized controlled trial. Am J Prev Med. 2012;43:527-32.

93. Centola D. Social media and the science of health behavior. Circulation. 2013;127:2135-44.

94. Valle CG, Tate DF, Mayer DK, et al. A randomized trial of a Facebook-based physical activity intervention for young adult cancer survivors. J Cancer Surviv. 2013;7:355-68.

95. Zhang J, Brackbill D, Yang S, Centola D. Efficacy and causal mechanism of an online social media intervention to increase physical activity: results of a randomized controlled trial. Prev Med Rep. 2015;2:651-7.

96. Duncan M, Vandelanotte C, Kolt GS, et al. Effectiveness of a web- and mobile phone-based intervention to promote physical activity and healthy eating in middle-aged males: randomized controlled trial of the ManUp Study. J Med Internet Res. 2014;16:e136.

97. Turner-McGrievy G, Tate D. Tweets, apps, and pods: results of the 6-month mobile pounds off digitally (mobile POD) randomized weight-loss intervention among adults. J Med Internet Res. 2011;13:e120.

98. Pope Z, Zeng N, Zhang R, et al. Effectiveness of combined smartwatch and social media intervention on breast cancer survivor health outcomes: a 10-week pilot randomized trial. J Clin Med. 2018;7:140.

99. Pope ZC, Barr-Anderson DJ, Lewis BA, et al. Use of wearable technology and social media to improve physical activity and dietary behaviors among college students: a 12-week randomized pilot study. Int J Environ Res Public Health. 2019;16.

100. Maher CA, Lewis LK, Ferrar K et al. Are health behavior change interventions that use online social networks effective? A systematic review. J Med Internet Res. 2014;16:e40.

101. Laranjo L, Arguel A, Neves AL, et al. The influence of social networking sites on health behavior change: a systematic review and meta-analysis. J Am Med Inform Assoc. 2015;22:243-56.

102. Tate DF, Jackvony EH, Wing RR. Effects of Internet behavioral counseling on weight loss in adults at risk for type 2 diabetes. JAMA, J Am Med Assoc. 2003;289:1833.
103. Morgan PJ, Lubans DR, Collins CE, et al. The SHED-IT randomized controlled trial: evaluation of an Internet-based weightloss program for men. Obesity. 2009;17:2025-32.

104. Turner-McGrievy GM, Campbell MK, Tate DF, et al. Pounds Off Digitally study: a randomized podcasting weight-loss intervention. Am J Prev Med. 2009;37:263-9.

105. Ballin M, Hult A, Bjork S, et al. Digital exercise interventions for improving measures of central obesity: a systematic review. Int J Public Health. 2020;65:593-605.

106. Lyzwinski LN. A systematic review and meta-analysis of mobile devices and weight loss with an intervention content analysis. J Pers Med. 2014;4:311-85.

107. Ryu B, Kim N, Heo E, et al. Impact of an electronic health record-integrated personal health record on patient participation in health care: development and randomized controlled trial of MyHealthKeeper. J Med Internet Res. 2017; 19:e401. Smartphone app linked to personal health record correlated with higher body weight loss but no change in PA in the short.

108. Loewenstein G, Asch DA, Volpp KG. Behavioral economics holds potential to deliver better results for patients, insurers, and employers. Health Aff (Millwood). 2013;32:1244-50.

109. Cadmus-Bertram L, Marcus BH, Patterson RE, et al. Use of the Fitbit to measure adherence to a physical activity intervention among overweight or obese, postmenopausal women: self-monitoring trajectory during 16 weeks. JMIR Mhealth Uhealth. 2015;3:e96.

110. Nicklas BJ, Gaukstern JE, Beavers KM, et al. Self-monitoring of spontaneous physical activity and sedentary behavior to prevent weight regain in older adults. Obesity (Silver Spring). 2014;22:1406-12.

111. Thomas JG, Raynor HA, Bond DS, et al. Weight loss in Weight Watchers Online with and without an activity tracking device compared to control: a randomized trial. Obesity (Silver Spring). 2017;25:1014-21.

112. Goldstein SP, Goldstein CM, Bond DS, et al. Associations between self-monitoring and weight change in behavioral weight loss interventions. Health Psychol. 2019;38:1128-36.

113. Morgan PJ, Lubans DR, Collins CE, et al. The SHED-IT randomized controlled trial: evaluation of an Internet-based weightloss program for men. Obesity (Silver Spring). 2009;17:2025-32.

Publisher's Note Springer Nature remains neutral with regard to jurisdictional claims in published maps and institutional affiliations. 